\title{
Degree of consistent training: Improvements in search performance and automatic process development
}

\author{
WALTER SCHNEIDER and ARTHUR D. FISK \\ University of Illinois, Champaign, Illinois 61820
}

\begin{abstract}
Previous research has shown substantial improvements in detection performance when subjects consistently detect a subset of stimuli. In contrast, in conditions in which stimuli appear as both targets and distractors, there is little performance improvement with practice. The present experiments examine how varying degrees of consistency determine the improvement of detection accuracy with extended practice. The degree of consistency was varied by manipulating the frequency with which a letter was a distractor while holding the number of occurrences as a target constant. The experiments utilized a multiple-frame target-detection search paradigm in which subjects were to detect single-letter targets in a series of rapidly presented letters on four channels. Experiments showed that detection performance improvement with practice was a monotonic function of the degree of consistency, decreasing to zero as the target-to-distractor ratio increased from 10:0 to 10:20. As consistency decreased, detection performance asymptoted earlier and at a lower level. A dual-task experiment examined subjects' ability to perform the previously trained search task as a secondary task. Results showed that the previous targetto-distractor consistency had a marked effect on resource sensitivity of the detection task. The general issues of consistency in the development of skilled performance and in the development of automatic processing are discussed.
\end{abstract}

Human performance in almost any cognitive skill improves with practice. But the amount of improvement is dramatically increased when subjects are able to deal consistently with their task. In detection paradigms, research has shown that extended practice at consistently attending to a subset of stimuli results in quantitative and qualitative changes in performance (e.g., Moray, 1959, 1975; Schneider \& Shiffrin, 1977). Reaction times are faster (e.g., Kristofferson, 1972a; Neisser, 1963; Schneider \& Shiffrin, 1977), detection performance is less affected by memory load (e.g., Logan, 1979; Neisser, 1963; Schneider \& Shiffrin, 1977) or number of channels (e.g., Duncan, 1980; Moray, 1975; Shiffrin, 1975), and performance becomes much less sensitive to attentional resource demands (LaBerge, 1973; Logan, 1979; Schneider \& Fisk, Note 1).

However, in conditions in which subjects cannot consistently attend to a subset of stimuli, extended practice results in little, if any, improvement in performance. Performance may improve somewhat due to familiarization with the task, understanding of instructions, etc., but, performance does not improve as profoundly as when stimuli are dealt with con-

This research was supported in part by funds from Office of Naval Research Personnel and Training Contract N000014-78-C0012 (NR 150-409) and NIMH Grant 5 R01 MH 3125. Reprint requests should be sent to Walter Schneider, Department of Psychology, University of Illinois, $603 \mathrm{E}$. Daniel, Champaign, Illinois 61820 . sistently. In both visual search (e.g., Kristofferson, 1972b; Rabbitt, 1978; Schneider \& Shiffrin, 1977) and detection paradigms (Schneider \& Shiffrin, 1977), subjects show little performance change after the first session.

Schneider and Shiffrin (1977) have emphasized that there are many quantitative and qualitative performance differences between tasks, depending on whether or not individual stimuli are consistently mapped. In a consistently mapped (CM) condition, given stimuli appeared only as targets and never as distractors for a given subject. Therefore, in the CM condition, subjects could consistently deal with the stimuli, because a CM stimulus was always a valid target which required a positive response. Subjects' performance in the $\mathrm{CM}$ condition showed dramatic improvement. In conditions not consistently mapped, a given character could be a target on one trial and a distractor on the next. These were referred to as varied mapping (VM) conditions by Schneider and Shiffrin (1977). In the VM conditions, subjects could not deal consistently with the stimuli because a VM stimulus sometimes required a positive response (i.e., a target) and sometimes had to be ignored (i.e., a distractor). The subjects' performance in the VM condition did not improve even after thousands of trials.

Visual search comparison slopes illustrate the large quantitative difference that can occur between CM and VM conditions. Schneider and Shiffrin (1977) 
found display comparison slopes ranging from about $3 \mathrm{msec}$ per item in the CM condition to $75 \mathrm{msec}$ in the VM condition (see Schneider \& Shiffrin, 1977, Figure 6, memory set size 4 , positive response). A transfer experiment showed a major qualitative difference in that $\mathbf{C M}$ training resulted in substantial negative transfer when the target and distractor set stimuli were reversed (Shiffrin \& Schneider, 1977, Experiment 1). However, there was no effect of changing stimuli in VM conditions (Shiffrin \& Schneider, 1977, Experiment 2). These two results illustrate only two of many differences between CM and VM conditions (for reviews, see Schneider, Dumais, \& Shiffrin, 1982; Shiffrin \& Schneider, 1977).

The profound changes in performance that occur when subjects consistently respond to stimuli have caused many researchers to suggest that there are two qualitatively different modes of human information processing (Hasher \& Zacks, 1979; James, 1890; LaBerge, 1973, 1975, 1976; Logan, 1978, 1979; Norman, 1976; Posner \& Snyder, 1975; Shiffrin \& Schneider, 1977). In this paper, we will refer to these two modes as control and automatic processing. Control processing is expected to occur in novel or varied mapping situations. Control processing requires little training to initiate and is easy to modify, but is slow, effortful, generally serial in nature and highly dependent on load (see Schneider, Dumais, \& Shiffrin, 1982; Shiffrin \& Schneider, 1977). Automatic processing occurs after extended practice in consistent mapping situations. Automatic processing is a relatively fast, effortless, parallel processing mode.

Consistently mapped training appears to be a necessary condition for improvements in the subjects' ability to timeshare tasks. Logan (1979) found that extended timesharing practice with a varied mapping search task and a short-term memory task did not eliminate the dual-task interaction. However, if the search stimuli were consistently mapped, extended practice eliminated the memory $\times$ search task interaction. Schneider and Fisk (Note 1) gave subjects extensive training at performing simultaneous $\mathrm{CM}$ and VM search. They found that with extended practice, performance in the consistently mapped condition became insensitive to the difficulty level of the varied mapping condition.

Although we know that varying degrees of consistency result in quite different performance characteristics, we do not know how performance declines as the degree of consistency is reduced. The purpose of the present experiments was to examine the effects of consistency on performance improvement in a detection paradigm. In particular, the experiments examined varying degrees of consistency by manipulating the frequency with which a letter appeared as a distractor while holding constant the number of appearances as a target. Detection improvement during
6,720 trials per subject was examined in Experiment 1. A dual-task paradigm was employed in Experiment 2 in order to examine the effect of degree of consistency in training on the ability of subjects to perform the search task as a secondary task.

\section{EXPERIMENT 1}

\section{Method}

Subjects. Five females and four males were paid for their participation in the present experiment. All subjects were students at the University of Illinois. One subject was left-handed.

Equipment. The experiment was controlled by a Digital Equipment Corporation PDP-11/34 computer. The computer was programmed to present the appropriate stimuli, collect responses, and control timing of the display presentation. The stimuli were presented on Tektronics Model 604 and Model 620 cathode ray scopes, which contained P-31 phosphors. Each subject wore a headset through which white noise $(80 \mathrm{~dB})$ and an error tone were carried.

Stimuli. The characters used in the present experiment were uppercase letters of the English alphabet. The characters were constructed from dots on a rectangular grid, 32 dots wide $\times 48$ dots high, with an average of $\mathbf{4 3}$ dots used to specify a character. The characters were $.52 \mathrm{deg}$ in width $\times .58 \mathrm{deg}$ in height. The refresh rate of the dots making up the stimuli was $10 \mathrm{msec}$. The display of the characters was divided into frames, with each frame consisting of four characters positioned to form a square around a center fixation dot. The distance from the focus dot to the center of each letter was $1 \mathrm{deg}$ of visual angle. The subjects' eyes were approximately $45 \mathrm{~cm}$ from the display. The characters used were: A, C, D, E, M, R, S, U, and Z. These letters were chosen (through pilot testing) such that each letter was approximately equally confusable with the other letters. The room was dimly lit $(.4 \mathrm{fc}$ incidental light), with each dot easily visible on the display (.005 $\mathrm{fL}$ per dot).

Trial sequence. Subjects searched for one letter (memory set size 1) in frames containing four letters. When the subjects detected the target letter, they pushed one of four buttons in a square indicating the position of the target.

Each trial consisted of the following sequence: the presentation of a memory set letter and accuracy feedback, a fixation dot, 12 $\rightarrow$ frames of letters, and either a "correct response" indicator or an error tone (see Figure 1). The memory set letter was presented in the upper left-hand corner of the scope. In addition, accuracy feedback was presented in this display and will be described below. The subjects were given up to $30 \mathrm{sec}$ to study the target item. The subjects initiated the frame sequence by pushing the initiate button with the index finger of the nonpreferred hand. After the initiation button was pushed, a fixation dot was presented for $500 \mathrm{msec}$. This provided a fixation point corresponding to the central fixation dot, which remained on for the entire frame sequence. The

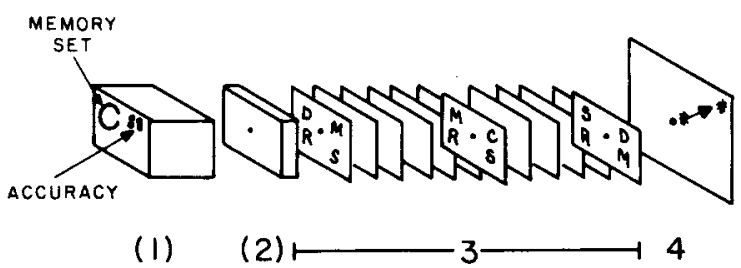

Figure 1. Trial sequence Experiment 1. (1) Memory set and accuracy feedback display. (2) Focus dot for .5 sec. (3) Twelve frames presented with target on Frame 7. (4) After a correct subject response, a mark spins off the screen from the target position. Note that masks presented between frames are not shown in the figure. 
frame sequence consisted of 12 frames presented in rapid succession. Each frame was composed of four letters presented for $80 \mathrm{msec}$, followed by four random dot masks (not depicted in Figure 1). The dot masks were presented for $30 \mathrm{msec}$ in the same display positions as the letters. These elements (letters and masks) were positioned to form a square around a center fixation dot. The display time of the letters plus the display time of the masks yielded a total frame time of $110 \mathrm{msec}$. For a given trial, a set of four distractor letters was used for all 12 frames. Hence, with the exception of the target frames, the same four letters appeared in different locations on each of the 12 frames. The letters were arranged randomly, with the restriction that no letter could appear in the same display position on two successive frames.

The target item was always presented once during the frame sequence. The target could occur during Frames 3-11. The target frame and the display location of the target within that frame were determined randomly. The subjects' task was to indicate the target's location by pushing one of four buttons with the preferred index finger. These buttons also formed a square and represented a one-to-one mapping of display position to response button. The subjects were instructed to guess the correct response at the end of the frame sequence if no target was detected.

Performance feedback was given to the subjects and consisted of two types. Error feedback consisted of a tone burst given through the subject's headset. The error feedback was given when the subject incorrectly indicated the target's display position. Accuracy feedback consisted of three separate types designed to increase subject motivation and reduce boredom. First, when the subject correctly indicated the target's location, a random dot pattern would appear to spin off the screen from the target's location (see Figure 1). Second, the subject's current within-block accuracy, indicated by a two-digit number, was presented along with the memory set display. Third, a "skill" rating, which corresponded to a given accuracy level, was given to the subject by blinking one of four colored LED lights on the subject's response box. Both the accuracy and the "skill" rating were initialized to zero at the beginning of each trial block.

The experiment was divided into two phases: training and test. A within-subject design was used for all experiments. The degree of consistency of a letter's appearing as a target or distractor in training blocks was the main independent variable. The frequency with which a given letter appeared as a target was held approximately equal, whereas the frequency of its appearing as a distractor varied. Let $(t: d)$ represent the number of times a given letter appears as a target $(t)$ vs. a distractor (d) in a block of trials. To illustrate, in Condition 10:20, a letter appeared as a target in 10 trials per block and as a distractor in 20 trials. When a letter appeared as a distractor, it appeared on almost all frames (i.e., it appeared on all frames except the target frame and had a .25 probability of being replaced on the target frame; hence, the letter appeared on an average of 11.75 frames). The consistency conditions run were 10:0,10:5, 10:10,10:20, and 9:61. In any of the first four conditions (i.e., 10:0 to 10:20), the distractors were selected from the 9:61 letters. When the letters from the 10:5, 10:10, and $10: 20$ conditions appeared as distractors, the targets were sampled from the 9:61 condition letters. One letter was assigned to each consistency condition, except for the 9:61 condition, which had five letters assigned to it. The letter assignment remained constant for each subject throughout this experiment and the next. The assignment of letters to conditions was counterbalanced across subjects, using a Latin square. The consistency conditions were manipulated between trials. During training, there were 85 trials per block (10 each of 10:0, 10:5, 10:10, and 10:20 conditions and 45 of the 9:61 - five target letters nine times each).

During the test phase, all letters except the ones assigned to the 9:61 condition were targets on 20 trials and never appeared as distractors. The 9:61 condition letters appeared with a test ratio of 4:52. There were 20 trials per letter per block in all conditions except the 4:52 condition, which had five letters each with 4 trials per block. The test blocks were run to assess detection accuracy in the 10:0 to 10:20 training condition during a block in which the stimuli appeared only as targets.

There were six training/test cycles, consisting of 12 training blocks of 85 trials followed by 1 test block of 100 trials. The subjects required 1050 -min sessions to complete the series.

Practice. Prior to participating in the experiments, the subjects were given 150 trials of practice to familiarize them with the experimental procedure. Only the letters subsequently used in the 9:61 condition appeared during practice. The selection of letters was such that the subjects had no exposure to any letters used subsequently in the other conditions (i.e., 10:0 through 10:20) in the experiment proper.

\section{Results and Discussion}

The data from the training part of the training/test cycles are presented in Figure 2. (The lines are not connected in this figure, indicating the occurrence of the intervening tests). The data are corrected for guessing in all cases (corrected probability equals probability of detection minus one-third the probability of an error). All reported accuracies in the 9:61 condition are for trials in which distractors were also chosen from the 9:61 condition. Detection performance when one of the 10:5, 10:10, and 10:20 letters appeared as a distractor was not different from trials when distractors were chosen only from the 9:61 conditions.

It is clear from Figure 2 that all of the conditions showed some improvement with time. The 10:0 and 10:5 conditions showed the largest detection improvement. The 10:20 and 9:61 conditions did not appear to differ substantially from each other throughout the experiment. Finally, the 10:10 condition generally maintained a "middle range" performance level throughout the experiment.

The data gathered during the intervening tests are plotted in Figure 3. Points are plotted as a function of the number of times a letter appears as a distractor during training. The data show the functional relationship between the degree of consistency and percent correct. The data show detection improvement in the completely consistent condition (10:0) relative

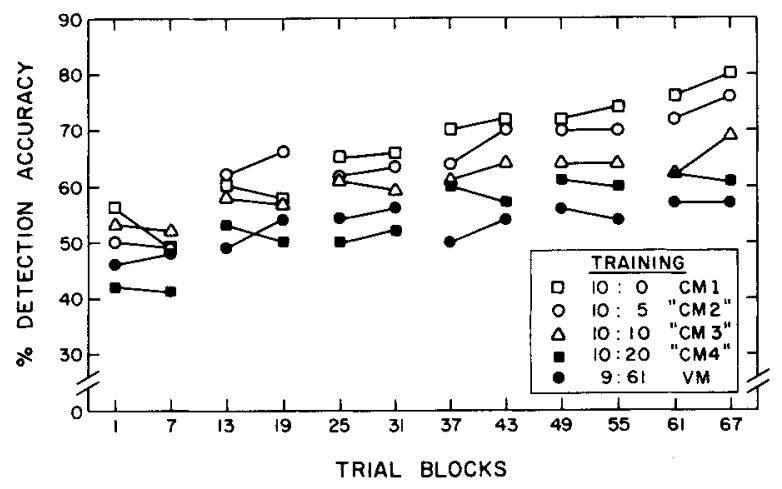

Figure 2. Experiment 1 training corrected detection accuracy as a function of training blocks (85 trials per block). After every 12th block of training, a test block occurred. Each point represents the data from six blocks. 


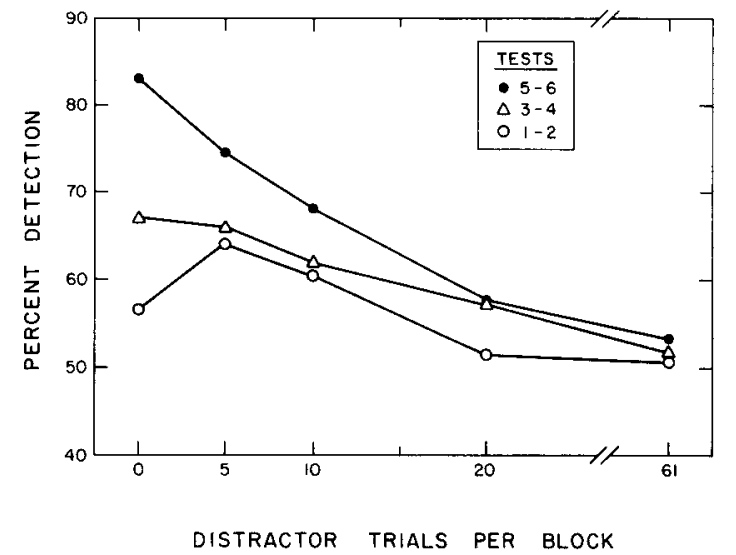

Figure 3. Experiment 1 corrected detection accuracy as a function of distractor frequency.

to other conditions. An analysis of variance [experimental conditions $\times$ time (intervening tests) $\times$ subjects] was performed on the transformed (arcsin) accuracy data. The main effects of experimental conditions and time reached statistical significance $[F(4,32)=4.1, p<.01$, and $F(5,40)=5.6, p<.001$, respectively]. The experimental condition $\times$ time interaction was also significant $[F(20,160)=1.9$, $p<.02]$. The analysis of simple main effects revealed substantial differences between conditions from Test 4 on ( $p<.001$ in all cases). Also, the only experimental condition significantly affected by time was the $10: 0$ condition $[F(5,40)=16.7, p<.0001]$. Post hoc comparisons ${ }^{1}$ of the averaged performance of Tests 5 and 6 between the conditions showed that only the 10:20 condition did not differ from the 9:61 condition $(F<1)$. The 10:0 condition differed statistically from both the $10: 10$ and 10:20 conditions. The 10:0 condition did not differ statistically from the $10: 5$ condition $(p=.11)$. The 10:5 condition did not differ from the 10:10 condition. The difference between the 10:5 and 10:20 conditions was significant $[F(1,8)=5.09, p=.05]$. Finally, the $10: 10$ condition did not differ from the 10:20 condition.

The present data suggest three conclusions. First, the more consistent the mapping, the greater the improvement with practice is. After 6,720 trials of practice, the 10:0 condition improved the most. Second, the rate of improvement is an increasing function of the degree of consistency. The improvement between tests (see Figure 3) appears to be an increasing function of consistency. A third tentative conclusion is that the greater the consistency, the lower and earlier the performance asymptote. The 9:61 condition appears to have asymptoted by the 19th training block (167 trials as a target letter) at about $52 \%$. The $10: 20$ condition appears to have asymptoted by Training Block 37 (420 trials per stimulus) at $57 \%$ (note, however, that this condition was not statistically different from the 9:61 condition). The other conditions are still clearly improving after 6,720 training trials (or 840 target trials per letter). Extreme amounts of training may be necessary before the $10: 0$ condition asymptotes. ${ }^{2}$

The present results agree with previous results in which VM conditions show early performance asymptotes. The present results also show that consistency has a graded effect rather than an all-or-none effect. The 10:10 condition improvement rate was about halfway between the 10:0 and 9:61 conditions (Tests 5 and 6).

\section{EXPERIMENT 2}

The results from Experiment 1 indicate that performance improvement is a function of consistency. The present experiment examines resource sensitivity as a function of the degree of consistency in training. Resource sensitivity refers to how much effort or attention is required to perform the task. Resource sensitivity is generally measured by how much performance declines when one task must be done simultaneously with another task (see Wickens, 1982). The resource sensitivity issue is important for two reasons. First, if processing in consistently mapped conditions can be done without cost of attentional (control process) resources, complex processing systems can be developed by building up automatic component processes (see Schneider, Dumais, \& Shiffrin, 1982). If practice in varied mapping conditions does not reduce the resource demands of the process, then tasks requiring multiple varied mapping processes will exceed resource capacity even after extended practice. This would preclude the development of complex processing systems that operate effectively when the component tasks are inconsistently mapped. In agreement with the above prediction, Schneider and Fisk (Note 1) have shown that subjects can perform a consistently mapped search task with no measurable decrement in resources. Also, performance in varied mapping conditions has still required attentional resources even after extended practice (Logan, 1979; Schneider \& Fisk, Note 1).

The second reason for interest in resource sensitivity is that it may be a defining characteristic of automatic and control processing. Logan (1979) has suggested that the lack of interaction between two conditions suggests the presence of an automatic process. Shiffrin, Dumais, and Schneider (1981) define one class of automatic processes as any process that does not decrease general, nonspecific processing capacity available to other processes. ${ }^{3}$ Hence, if decreasing consistency reduces automatic process development, secondary task performance should be a decreasing function of the degree of consistency.

The present experiment examined the ability of subjects, who were trained under varying degrees of consistency in Experiment 1, to detect targets while 
concurrently performing a VM detection task. The subjects were required to treat the VM detection task as the primary task.

\section{Method}

Subjects. The subjects who participated in the previous experiment were employed in Experiment 2. Sessions in Experiment 2 were scheduled to be contiguous with Experiment 1, with $1 \mathrm{~h}$ to 2 days between sessions.

Procedure. The present experiment used the stimuli that had been used in the previous experiment. All previously used items except the 9:61 conditions were consistently mapped in the present experiment. As in the previous experiment, only one target could occur in each trial. For purposes of discussion, the previous conditions of 10:0, 10:5, 10:10, and 10:20 will be referred to as consistently mapped (CM) letters and the previous 9:61 condition will be referred to as variedly mapped (VM) letters. On a per block basis, CM letters appeared with a target-to-distractor ratio of 8:0 and the VM letters with a ratio of 5.6:32.6.

There were four basic conditions in this experiment: (1) Single target (VM search)-one memory set item from the VM letters (9:61) was presented, and the subject's task was to detect this item in the upcoming frame sequence. (2) Single target CM searchthe subject searched for one memory set item from the CM set (10:0 to 10:20). (3) Dual VM target (dual task)-two VM letters were presented in the memory set display, with one of these letters occurring as a target in the frame sequence. (4) Dual VM/CM (dual task)-in this condition the memory set display contained one VM letter and a dot mask to the right of the VM letter. This indicated to the subjects that the VM letter might appear or that any one of the four previously trained on CM letters might occur during the frame sequence. For this condition, the subjects were instructed to protect their VM performance. They were told, "If you were being paid based upon your detection accuracy, you would be paid conditional on your VM accuracy in this condition." In the dual VM/CM condition, only VM performance entered into the performance feedback. This was done to clearly emphasize the VM search. For the dual VM/CM trials, VM targets occurred $50 \%$ of the time, while each CM letter occurred on $12.5 \%$ of the trials. Each block of trials contained $32 \mathrm{VM} / \mathrm{CM}$ trials, 16 of which contained the VM target item with 4 trials allocated to each CM target. During the first five blocks of trials, the subjects were given a card containing the four $\mathrm{CM}$ items.

For the single-task condition, accuracy feedback was given to the subjects as in Experiment 1. For the dual-task trials, the VM average accuracy was displayed below and to the right of the rightmost memory set character. The separation between the rightmost memory set character and the leftmost number of the accuracy was approximately $2 \mathrm{deg}$.

The experimental conditions were manipulated between trials. There were 60 trials per block. Each of four CM letters occurred four times as a single-task target and four times as a dual-task target. Each of five VM letters occurred as targets across 4 VM single-task trials, $16 \mathrm{VM}$ target trials in the $\mathrm{VM} / \mathrm{CM}$ condition, and $8 \mathrm{VM}(\mathrm{M}=2)$ trials. Due to a limitation of number of characters for the VM $(M=2)$ condition, frame size was reduced to three for all conditions. A random-dot mask was used as a "place holder," and its display location was determined randomly for each frame. There were 40 blocks run over an average of four sessions.

\section{Results and Discussion}

Figure 4 presents the data from Experiment 2. This figure plots performances as a function of how frequently a given letter appeared in the $\mathrm{CM}$ training condition $(10: 0,10: 5,10: 10,10: 20)$ and the relevant VM condition. The VM conditions (trained in the 9:61 condition) are also plotted. Performance in the

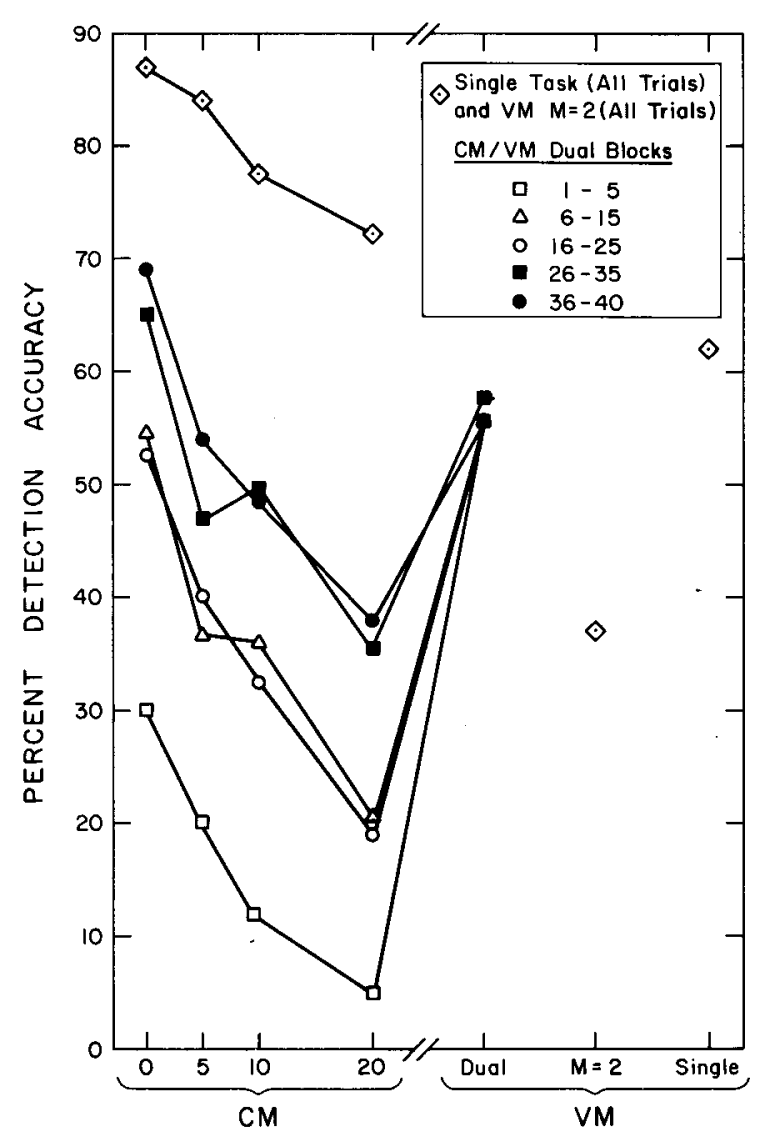

Figure 4. Experiment 2 single- and dual-task performance as a function of frequency as a distractor. $\mathrm{CM}$ letters never appeared as distractors in this experiment. The $\mathrm{CM}$ conditions are plotted as a function of frequency of occurrence as a distractor during previous CM training (see Figure 3).

single conditions (open diamonds in Figure 4) was averaged over the entire experiment, producing one point per single condition. The dual-task conditions are plotted to show the family of curves created as subjects performed the dual tasks better.

Figure 4 shows that the previous consistency of training had a marked and long-term effect on detection performance in the present experiment. The single-task CM condition (diamonds, upper left Figure 4) show a $15 \%$ reduction in detection accuracy as a function of previous training consistency (10:0 to 10:20). The dual-task CM conditions (lower left, Figure 4) show a $30 \%$ effect of training consistency which remained throughout the 40 blocks $(800 \mathrm{CM}$ target presentations per letter). Dual-task CM accuracy improved greatly $(39 \%)$ with practice, whereas the VM tasks were unaffected by practice. There was a small $(5 \%)$, nonsignificant difference between VM single (right, Figure 4) and VM dual (center, Figure 4) performance. This nonsignificant difference indicates that subjects maintained single-task VM performance levels when shifting from the single- to the dual-task conditions. The $25 \%$ drop between the VM 
single and $\mathrm{VM} M=2$ condition (lower right, Figure 4) indicates that subjects could not maintain VM performance in dual-task VM search.

An analysis of variance (experimental condition $x$ time $\times$ subjects) was performed and revealed a significant main effect of experimental conditions $[F(10,80)=13.2, p<.001]$ and a significant main effect of time (trial blocks in groups of five) $[F(7,56)$ $=9.16, p<.0011$. The experimental condition $\times$ time interaction was also significant $[F(70,560)=2.0$, $\mathrm{p}<.001]$. An analysis of the simple main effects found that, for the single-task conditions, only the previous $10: 10$ condition changed over time $[F(7,56)$ $=2.8, \mathrm{p}<.02 \mathrm{]}$. In addition, all $\mathrm{CM}$ dual-task conditions improved over time ( $\mathrm{p}<.001$ in all cases). The VM dual conditions [i.e., VM $(M=2)$ and VM in the $\mathrm{VM} / \mathrm{CM}$ condition] remained stable throughout the experiment ( $F<1$ in both cases). The dual-task VM performance was not significantly different from the single VM search condition $(p>.05)$.

The results show that consistent training greatly enhances subjects' ability to perform the detection task when it is a secondary task. The effect of previous degree of consistency in training is clearly evident in all dual-task CM conditions in Figure 4. The dual-task CM accuracy for the 10:0 condition averaged $30 \%$ above the 10:20 condition: The more consistent the previous training, the better the performance. The small nonsignificant decrement $(5 \%)$ in the VM dual vs. VM single condition suggest that subjects were allocating few, if any, resources to the $\mathrm{CM}$ conditions. The large decrement $(25 \%)$ from VM single to memory set size 2 indicates that the VM task was resource limited. Hence, any substantial reallocation of resources from the VM task would be expected to significantly reduce the VM single performance. We conclude that the $\mathrm{CM}$ dual condition represents performance with few, or no, resources allocated to the CM task.

In all dual-task conditions, $\mathrm{CM}$ performance improves with practice. There are two potential reasons for this improvement. First, subjects may need experience with dual-task situations to enable effective performance. Since the 10:0 condition received 840 training trials during Experiment 1 and only 160 trials during Experiment 2, it is unlikely that the $39 \%$ improvement in dual-task performance (between Blocks 1-5 and 36-40) was due to additional CM training alone. We believe that subjects were learning to integrate the $\mathrm{CM}$ and $\mathrm{VM}$ searches. In a series of dual-task experiments, Schneider and Fisk (Note 1) found that subjects typically required $2 \mathrm{~h}$ of training to integrate two single well-practiced tasks.

The second possible reason for improvement is the benefit of purely consistent practice in the previously low degree of consistency conditions $(10: 10,10: 20)$. Since the $(10: 20)$ condition did not show significant improvement with practice in Experiment 1, training in the present experiment was the first opportunity for consistent practice leading to improved performance.

We would expect subjects to perform the dual-task $\mathrm{CM} / \mathrm{VM}$ as well as they performed the individual tasks, given sufficient practice. The subjects' dualtask CM performance is clearly improving throughout the present experiment. Schneider and Fisk (Note 1) report that, given sufficient training, subjects were able to perform a simultaneous CM/VM task without a sensitivity deficit to either task. These results indicate that subjects can perform CM tasks using few, or no, resources.

Performance for the first five blocks in the 10:20 conditions was effectively at chance $(5 \%)$ detection accuracy. The subjects could not detect the previous 10:20 letters when that detection was a secondary task. Hence, within an inconsistently mapped condition, practice appears not to develop automatic, nonresource consumptive processing.

The present experiment has been effective in showing differential dual-task detection ability across the previously trained $\mathrm{CM}$ conditions: The higher the consistency during training, the better the performance in dual-task conditions.

\section{GENERAL DISCUSSION}

The present experiments have demonstrated that detection accuracy in a visual search task is a monotonically increasing function of consistency. Experiment 1 showed that little, if any, improvement occurs with practice when the probability of a stimulus's being a target or distractor is equal.

Practice does not improve performance. Only consistent practice improves performance. Practice and consistency appear to have a multiplicative effect on performance improvement. Large amounts of practice in the 9:61 condition produced little improvement in performance (Figure 3). Also, consistency shows little benefit until a substantial number of practice trials have occurred. There was no clear benefit for consistency with only 560 training trials in Experiment 1.

The reader should be cautious about generalization when using the degree of consistency figures presented in these experiments. We did not find performance improvement when a letter appeared as a target on 10 trials and as a distractor on 20. But note that if a letter appeared as a distractor, it appeared on an average of 11.75 frames. Hence, it is not clear whether the degree of consistency for no improvement should be represented as 10:20 or as 10:235. We feel that the effect of degree of consistency will be determined by the relationship between times a stimulus is detected as a target vs. the number of times it is actively examined (through control processing) and discarded. The number of active ex- 
aminations will be very dependent on the type of task involved, the type of display, presentation time, etc.

The ability to perform the detection task as a secondary task is critically dependent on the degree of consistency in training. In the early blocks of the dual-task experiment, the previous $10: 10$ and 10:20 conditions were near chance $(12 \%$ and $5 \%$, respectively, Blocks 1-5). This suggests that the 10:10 and 10:20 conditions in Experiment 1 did not enable subjects to detect targets without allocating attention to the detection task.

The present results support initial attempts to define automatic processing in terms of resource costs. There is at present no agreed-upon set of necessary and sufficient properties to define an automatic process (see Shiffrin, Dumais, \& Schneider, 1981). Two characteristics commonly associated with automatic processing are that subjects' performance improves with extended training and that subjects can perform automatic tasks with few, or no, resources allocated to them. In high-consistency conditions, performance improved with practice (Experiment 1) and subjects could detect $\mathrm{CM}$ targets without any significant reduction in simultaneous VM search (Experiment 2).

The results suggest that consistency is a necessary condition for automatic process development. Performance improvement and resource sensitivity were functions of the amount and consistency of practice. In the 10:20 conditions, performance did not improve with practice, nor could the subjects perform the task under high secondary task load. It is important to note that the 10:20 and 10:0 conditions were searched for an equal number of times and that the 10:20 conditions received about $82 \%$ as many correct detections during training as did the 10:0 condition. Still, performance did not improve in the 10:20 condition. The results suggest that consistency is a necessary condition for automatic process development (this is in agreement with Logan, 1979). ${ }^{4}$

The results support the view that automatic processing is a graded rather than all-or-none process. The detection performance was a graded function of consistency; individual subject curves show graded functions similar to those of the group data. (The data also support a view that the ability of the automatic process to operate in parallel with simultaneous control processing is a graded function of consistency.) The subject detects a target when either process detects a target. Evidence for such parallel automatic and control processing is presented by Ellis and Chase (1971; also discussed in detail by Schneider \& Shiffrin, 1977, p. 40) for a consistently mapped letter-size judgment and a variably mapped memory search task.

The results suggest that automatic processing performance can generalize to situations with less than perfect consistency. The benefits of automatic pro- cessing (e.g., greater speed, parallel processing, less effort, nonresource consumptive) should occur in processes that have a high-probability outcome as well as processes that have a perfectly consistent outcome.

The degree of consistency appears to be a central concept in the learning literature. In concept learning, inconsistent feedback (referred to as "misinformation") results effectively in no learning when "misinformation" occurs on over 30\% of the trials (Pishkin, 1960; Rogers \& Haygood, 1968). Exposure to random (inconsistent) reinforcement can slow the learner considerably in concept formation (Trabasso \& Staudenmayer, 1968). Cue differentiation in multiplecue discrimination learning is a function of cue validity (Friedman, Trabasso, \& Mosberg, 1967). In singlecue probability learning, subjects fail to identify consistent relationships when stimuli show low $(\mathrm{r}<.30)$ correlations to criterion responses (Brehmer, 1978; Johansson \& Brehmer, 1979). In free recall, the concept of subjective organization (Tulving, 1966) suggests that learning is a function of the degree to which subjects can organize the list (see Crowder, 1976, for discussion). In digit series learning, a lack of consistency of grouping results in no transfer between list repetitions (Bower \& Winzenz, 1969; Johnson \& Migdoll, 1971). In animal discrimination learning, the probability of attending to a stimulus dimension is a function of the consistency with which attending to that dimension results in consistent reinforcement (Mackintosh \& Holgate, 1968; Sutherland, 1964).

Learning a complex skill, such as reading, seems to be influenced by consistency. Illiteracy rates in developed countries suggest the importance of consistency in learning to read. Bouwhuis (Note 2) reports that illiteracy in English-speaking countries (United States, Canada, and England) is about twice that of non-English-speaking countries with comparable levels of industrial development and commitment to education (Germany, Denmark, Belgium, Netherlands, Spain, Italy). Bouwhuis suggests that this difference is due to the lack of phonetic consistency of English. Training using the i.t.a. (Initial Teaching Alphabet) method teaches children initially to read in the i.t.a. alphabet, which maps phonemes to orthographic symbols with perfect consistency. Training students to first read a perfectly consistent alphabet substantially increases the acquisition of reading and writing and reduces the incidence of reading disability to about half that seen with traditional orthography (Downing, 1969; Note 3). ${ }^{5}$ The present work shows that, as consistency decreases, the effectiveness of automatic processing decreases. Hence, users of phonetically inconsistent languages should have more difficulty becoming automatic word encoders.

These results all show learning to be not a function of executions, but rather a function of consistent executions. Automatic processing develops when sub- 
jects deal with information in a consistent manner. As the degree of consistency declines, the effectiveness of automatic processing declines, and control processing becomes necessary.

William James (1890) provided an excellent metaphor specifying consistency as essential for developing automatic habits. He stated (p. 123):

Never suffer an exception to occur till the new habit is securely rooted in your life. Each lapse is like the letting fall of a ball of string which one is carefully winding up; a single slip undoes more than a great many turns will wind again. Continuity of training is the great means of making the nervous system act infallibly right.

\section{REFERENCE NOTES}

1. Schneider, W., \& Fisk, A. D. Dual task automatic and control visual search: Can processing occur without resource cost? Manuscript submitted for publication, 1981.

2. Bouwhuis, D. Personal communication, April 1980. Some of the relevant data are included in his doctoral dissertation, "Visual Recognition of Words," University of Nijmegen, 1979.

3. Downing, J. The effectiveness of i.t.a. (Initial Teaching Alphabet) in the prevention and treatment of dyslexia and dysgraphia. Paper presented at the World Mental Health Assembly, Washington, D.C., November 1969.

\section{REFERENCES}

Bower, G. H., \& Winzenz, D. Group structure, coding, and memory for digit series. Journal of Experimental Psychology Monograph, 1969, 82(2, Pt. 2).

Brenmer, B. Response consistency in probabilistic inference tasks. Organizational Behavior and Human Performance, 1978, 22, 103-115.

Crowde R, R. G. Principles of learning and memory. Hillsdale, N.J: Erlbaum, 1976.

Downing, J. New experimental evidence of the effectiveness of i.t.a. in preventing disabilities of reading and spelling. Developmental Medicine and Child Neurology, 1969, 11, 547-555.

Duncan, J. The locus of interference in the perception of simultaneous stimuli. Psychological Review, 1980, 87, 272-300.

Eluis, S. H., \& Chase, W. G. Parallel processing in item recognition. Perception \& Psychophysics, 1971, 10, 379-384.

Friedman, M. P., Trabasso, T. R., \& Mosberg, L. Tests of a mixed model for paired-associates learning with overlapping stimuli. Journal of Mathematical Psychology, 1967, 4, 316-334.

Hasher, L., \& Zacks, R. T. Automatic and effortful processes in memory. Journal of Experimental Psychology: General, 1979, 108, 356-388.

JAmes, W. Principles of psychology (Vol. 1). New York: Holt, 1890.

Johansson, R., \& Brehmen, B. Inferences from incomplete information-A note. Organizational Behavior and Human Performance, 1979, 24, 141-145.

Johnson, N. F., \& Migdoll, D. M. Transfer and retroaction under conditions of changed organization. Cognitive Psychology, 1971, 2, 229-237.

Kristoffenson, M. W. Effects of practice on character classification performance. Canadian Journal of Psychology, 1972, 26, 54-60. (a)

KRISTOFFERSON, M. W. When item recognition and visual search functions are similar. Perception \& Psychophysics, 1972, 12, 379-384. (b)
LABERGE, D. Attention and the measurement of perceptual learning. Memory \& Cognition, 1973, 1, 268-276.

LABERGE, D. Acquisition of automatic processing in perceptual and associative learning. In P. M. A. Rabbitt \& S. Dornic (Eds.), Attention and performance V. New York: Academic Press, 1975.

LABerge, D. Perceptual learning and attention. In W. K. Estes (Ed.), Handbook of learning and cognitive processes (Vol. 4). Hillsdale, N.J: Erlbaum, 1976.

Logan, G. D. Attention in character-classification tasks: Evidence for the automaticity of component stages. Journal of Experimental Psychology: General, 1978, 107, 32-63.

LogAN, G. D. On the use of a concurrent memory load to measure attention and automaticity. Journal of Experimental Psychology: Human Perception and Performance, 1979, 5, 189-207.

Mackintosh, N. J., \& Holgate, V. Effects of inconsistent reinforcement on reversal and nonreversal shifts. Journal of Experimental Psychology, 1968, 76, 154-159.

Moray, N. Attention in dichotic listening: Affective cues and the influence of instructions. Quarterly Journal of Experimental Psychology, 1959, 11, 56-60.

Moray, N. A data base for theories of selective listening. In P. M. A. Rabbitt \& S. Dornic (Eds.), Attention and performance $V$. New York: Academic Press, 1975.

Neisser, U. Decision time without reaction time: Experiments in visual scanning. American Journal of Psychology, 1963, 76, 376-385.

Norman, D. A. Memory and attention: An introduction to human information processing (2nd ed.). New York: Wiley, 1976.

Pishkin, V. Effects of probability of misinformation and number of irrelevant dimensions upon concept identification. Journal of Experimental Psychology, 1960, 59, 371-378.

Posmer, M. I., \& S Syder, C. R. R. Attention and cognitive control. In R. L. Solso (Ed.), Information processing and cognition: The Loyola Symposium. Hillsdale, N.J: Erlbaum, 1975.

RABBItT, P. Sorting, categorization, and visual search. In E. C. Carterette \& M. P. Friedman (Eds.), Handbook of perception. New York: Academic Press, 1978.

Rogens, S. P., \& HaYgood, R. C. Hypothesis behavior in a concept-learning task with probabilistic feedback. Journal of Experimental Psychology, 1968, 76, 160-165.

Schneider, W., Dumars, S. T., \& Shiffrin, R. M. Automatic and control processing and attention. In R. Parasuraman, R. Davis, \& J. Beathy (Eds.), Varieties of attention. New York: Academic Press, 1982.

Schneider, W., \& Shiffrin, R. M. Controlled and automatic human information processing: $I$. Detection, search and attention. Psychological Review, 1977, 84, 1-66.

Shifrrin, R. M. The locus and role of attention in memory systems. In P. M. A. Rabbitt \& S. Dornic (Eds.), Attention and performance $V$. New York: Academic Press, 1975.

Shiffrin, R. M., Dumais, S. T., \& Schneider, W. Characteristics of automatism. In J. B. Long \& A. D. Baddeley (Eds.), Attention and performance IX. Hillsdale, N.J: Erlbaum, 1981.

Shiffrin, R. M., \& Schneider, W. Controlled and automatic human information processing: II. Perceptual learning, automatic attending and a general theory. Psychological Review, $1977,84,127-190$.

Sutherland, N. S. The learning of discrimination by animals. Endeavour, 1964, 23, 148-152.

Trabasso, T., \& Staudenmayer, H. Random reinforcement in concept identification. Journal of Experimental Psychology, $1968,77,447-452$.

Tulvina, E. Subjective organization and effects of repetition in multi-trial free-recall learning. Journal of Verbal Learning and Verbal Behavior, 1966, 5, 193-197.

Wickens, C. D. Processing resources in attention. In $R$. Parasuraman, R. Davis, \& J. Beathy (Eds.), Varieties of attention. New York: Academic Press, 1982. 


\section{NOTES}

1. The $F$ ratios for the significant comparisons were: 10:0-9:61, $F(1,8)=23.2 ; 10: 5-9: 61, F(1,8)=35.3 ; 10: 10-9: 61, F(1,8)=26.4$. $F$ ratios for 10:0-10:10 and 10:0-10:20 comparisons were: $F(1,8)=$ 6.2 and $F(1,8)=19.2$, respectively.

2. Shiffrin and Schneider (1977, Experiment 4c) found that subjects' performance in $\mathrm{CM}$ conditions was still improving after 20,000 training trials and was probably being limited more by retinal integration (frame time was down to $30 \mathrm{msec}$ ) than by more central comparison processing.

3. Shiffrin, Dumais, and Schneider (1981) also propose a second class of automatic processes as any process that demands resources in response to external stimulus input, regardless of the subject's attempts to ignore the distraction.
4. It should be noted that we have not defined what the consistent feature is. In the present experiment, the consistent feature was a letter shape. It might be possible to train automatic detection to a more global feature (e.g., difference in color between target and background) even though the elemental features (e.g., specific colors) are not consistently mapped.

5. Note that children seem to have little difficulty transferring from i.t.a. to traditional orthography in about 6 months. After the transition is complete, i.t.a. students read better than controls (Downing, Note 3).

(Manuscript received December 22, 1980; revision accepted for publication October 21, 1981.) 